\title{
Electroproduction of Majorana neutrinos
}

\author{
W. Buchmüller and C. Greub \\ Deutsches Elektronen-Synchrotron DESY, W-2000 Hamburg, FRG \\ Received 16 November 1990
}

\begin{abstract}
In the standard model with right-handed neutrinos the charged weak current in general contains light and heavy Majorana neutrinos. We calculate the production cross section for heavy neutrinos in electron-proton scattering and also the transverse momentum spectrum of the final state leptons. At HERA Majorana neutrinos with masses up to $180 \mathrm{GeV}$ can be produced for charged current couplings consistent with universality.
\end{abstract}

Right-handed neutrinos [1] are predicted by most extensions of the standard model. They appear in leftright symmetric theories, in models with mirror fermions or in unified theories based on the gauge group $\mathrm{SO}(10)$. If the known neutrinos are massive one has to understand why they are so much lighter than other leptons and quarks. Right-handed neutrinos can provide a natural explanation for very small Majorana masses of $v_{\mathrm{e}}, v_{\mu}$ and $v_{\tau}$ via the see-saw mechanism [2].

Also the symmetry structure of the standard model points towards the existence of right-handed neutrinos. The two global $\mathrm{U}(1)$ symmetries baryon number $(B)$ and lepton number $(L)$ are not separately conserved [3], because of the Adler-Bell-Jackiw anomaly. However, the difference $B-L$ is conserved, which suggests that this symmetry may be gauged in a more fundamental theory which contains the standard model as low-energy effective theory. If one then requires anomaly freedom for all gauge interactions including gravity [4], the trace of $B-L$ has to be the same for left-and right-handed fermions. This is indeed the case if right-handed neutrinos are added to the fermions of the standard model.

In contrast to all other leptons and quarks right-handed neutrinos have no strong and electroweak gauge interactions. Hence Majorana mass terms are allowed, and the most general renormalizable lagrangian for neutrinos and their interactions reads

$$
\mathscr{L}_{v}=\bar{\ell} \mathrm{i} \not \bar{\ell} \ell+\overline{\nu_{\mathrm{R}}} \mathrm{i} \not \partial \nu_{\mathrm{R}}-\bar{\ell} \phi g_{\mathrm{v}} \nu_{\mathrm{R}}-\overline{\nu_{\mathrm{R}}} g_{\mathrm{v}}^{\dagger} \phi^{\dagger} \ell-\frac{1}{2}\left(\overline{\nu_{\mathrm{R}}} m \nu_{\mathrm{R}}^{\mathrm{c}}+\overline{\nu_{\mathrm{R}}^{\mathrm{c}}} m^{\dagger} \nu_{\mathrm{R}}\right) \text {. }
$$

Here $\ell=\left(\nu_{\mathrm{L}}, e_{\mathrm{L}}^{-}\right)$is the lepton doublet, $\phi$ is the doublet of Higgs fields, $\mathrm{D}_{\mu}$ is the gauge invariant derivative, $\nu_{\mathrm{R}}^{\mathrm{c}}=C \overline{\nu_{\mathrm{R}}}, g_{v}$ and $m$ are $3 \times 3$ matrices of Yukawa couplings and Majorana masses for three quark-lepton generations. The Majorana mass matrix $m$ can always be chosen diagonal and real.

Spontaneous symmetry breaking of the electroweak gauge group generates the Dirac neutrino mass matrix $m_{\mathrm{D}}=g_{v} v$, where $v=\langle\phi\rangle_{0}=174 \mathrm{GeV}$. Assuming that the eigenvalues of $m$ are large compared to the elements of $m_{\mathrm{D}}$, the entire mass matrix can be diagonalized in a power series in $m^{-1} m_{\mathrm{D}}$. For a proper choice of the fields $\nu_{L}$ the mass eigenstates are then given by (cf. ref. [5]):

$\nu=L+L^{\mathrm{c}}, \quad N=R+R^{\mathrm{c}}$,

where

$\nu_{\mathrm{L}}=L+m_{\mathrm{D}}(1 / m) R^{\mathrm{c}}-\frac{1}{2} m_{\mathrm{D}}\left(1 / m^{2}\right) m_{\mathrm{D}}^{\dagger} L+\ldots$, 


$$
\begin{aligned}
\nu_{\mathrm{R}} & =R-(1 / m) m_{\mathrm{D}}^{\mathrm{T}} L^{\mathrm{c}}-\frac{1}{2}(1 / m) m_{\mathrm{D}}^{\mathrm{T}} m_{\mathrm{D}}^{*}(1 / m) R \\
& +\frac{1}{2}(1 / m)\left[m_{\mathrm{D}}^{\mathrm{T}} m_{\mathrm{D}}^{*}(1 / m)+(1 / m) m_{\mathrm{D}}^{*} m_{\mathrm{D}}\right](1 / m) m_{\mathrm{D}}^{\mathrm{T}} L^{\mathrm{c}}+\ldots,
\end{aligned}
$$

and

$\mathscr{L}_{v}=\frac{1}{2} \bar{\nu}\left(\mathrm{i} \not{\phi}-m_{v}\right) \nu+\frac{1}{2} \bar{N}\left(\mathrm{i} \not{\phi}-m_{\mathrm{N}}\right) N+\mathrm{O}\left(1 / m^{3}\right)$,

with

$m_{\mathrm{N}}=m+\mathrm{O}(1 / m)$

$m_{\mathrm{v}}=-m_{\mathrm{D}}(1 / m) m_{\mathrm{D}}^{\mathrm{T}}+\mathrm{O}\left(1 / m^{3}\right)$,

where, to leading order in $1 / m, m_{\mathrm{N}}$ and $m_{\mathrm{v}}$ are both diagonal and real. The light neutrino masses, i.e. the eigenvalues of $m_{\mathrm{v}}$, have to satisfy the experimental upper bounds [6] $m_{\mathrm{ve}_{\mathrm{e}}}<18 \mathrm{eV}, m_{\mathrm{v}_{\mu}}<0.25 \mathrm{MeV}$ and $m_{\mathrm{v}_{\mathrm{\tau}}}<35$ $\mathrm{MeV}$. Within the standard model one may expect that the heavy neutrino masses are of the order of the Higgs vacuum expectation value, i.e. $m=\mathrm{O}(v)$. In fact, in some models with dilatons, they are not allowed to be much heavier, and the same mechanism which dynamically determines the vacuum expectation value $v$, also yields Majorana neutrino masses of the same order of magnitude [5].

It is intriguing that heavy Majorana masses of order $v$ are sufficient to explain the smallness of the light neutrino masses via the see-saw formula (7). From $\operatorname{det}(m)=v^{3}$, eq. (7) and the experimental upper bounds one finds $\operatorname{det}\left(m_{\mathrm{D}}\right)<9 \times 10^{-4} \mathrm{GeV}^{3}$. For comparison one has for charged leptons, down- and up-type quarks: $\operatorname{det}\left(m_{\ell}\right)=9 \times 10^{-3} \mathrm{GeV}^{3}, \operatorname{det}\left(m_{\mathrm{d}}\right) \approx 7 \times 10^{-3} \mathrm{GeV}^{3}$ and $\operatorname{det}\left(m_{\mathrm{u}}\right)>5 \times 10^{-1} \mathrm{GeV}^{3}$ for $m_{\mathrm{t}}>89 \mathrm{GeV}$. Hence, no "unnaturally" small Dirac neutrino masses are needed in order to satisfy the stringent experimental upper bounds on light neutrino masses. Of course, it is also conceivable that the Majorana mass matrix $m$ is determined by a new interaction scale $A$ which is larger than the Fermi scale $v$. In left-right symmetric models, for instance, the masses of the new gauge bosons $W_{\mathrm{R}}$ and the Majorana neutrinos have to satisfy severe constraints [7] which do not allow sufficiently large cross sections for the production of heavy neutrinos at HERA [8].

In order to compute production cross sections for heavy neutrinos within the standard model one has to know the charged and neutral weak currents in terms of the mass eigenstates $\nu_{i}$ and $N_{i}$. Using eqs. (2)-(4) one obtains:

$$
\begin{aligned}
& J_{\mathrm{NC}}^{\mu}=\frac{g}{2 \cos \theta_{\mathrm{w}}}\left[\nu\left(1-m_{\mathrm{D}} \frac{1}{m^{2}} m_{\mathrm{b}}^{\ddagger}\right) \gamma^{\mu} \frac{1-\gamma_{5}}{2} \nu-\bar{N} \frac{1}{m} m_{\mathrm{b}}^{\ddagger} m_{\mathrm{D}} \frac{1}{m} \gamma^{\mu} \frac{1-\gamma_{5}}{2} N+\bar{N} \frac{1}{m} m{ }^{\ddagger} \gamma^{\mu} \frac{1-\gamma_{5}}{2} \nu\right. \\
& \left.+\bar{\nu} m_{\mathrm{D}} \frac{1}{m} \gamma^{\mu} \frac{1-\gamma_{\mathrm{S}}}{2} N+\mathrm{O}\left(1 / m^{3}\right)\right], \\
& J_{\mathrm{C} C}^{\mu}=\frac{g}{\sqrt{2}}\left[\bar{e} V\left(1-\frac{1}{2} m_{\mathrm{D}} \frac{1}{m^{2}} m_{\mathrm{D}}^{\ddagger}\right) \gamma^{\mu} \frac{1-\gamma_{5}}{2} \nu+\bar{e} V m_{\mathrm{D}} \frac{1}{m} \gamma^{\mu} \frac{1-\gamma_{5}}{2} N+\mathrm{O}\left(1 / m^{3}\right)\right] .
\end{aligned}
$$

Here $V$ is a Kobayashi-Maskawa type matrix for leptons. The upper bound on the matrix elements $\left|V_{\mathrm{e} i}\right|^{2}, i=\mathrm{e}$, $\mu, \tau$, decreases from 1 to $10^{-5}$ if the masses $m_{v_{i}}$ increase from 0 to $30 \mathrm{MeV}$ [9]. The matrix elements of $\xi=$ $m_{\mathrm{D}}(1 / \mathrm{m})$, which determine the electron couplings to heavy neutrinos, are constrained by the universality of the charged current which yields $\xi_{e i}^{2}<0.02[10]$. Such rather large mixings of order $10^{-2}$ are indeed compatible with the experimental upper bounds on light neutrino masses. This requires matrices $m_{\mathrm{D}}$ and $m$, which cannot be simultaneously diagonalized, and furthermore large ratios between different elements of $m_{\mathrm{D}}$ as they also occur in the mass matrices of charged leptons and quarks. Note, that for diagonal matrices $m_{\mathrm{D}}$ and $m$ one would obtain much smaller mixings, e.g., $\xi_{\mathrm{e} i}=\xi_{\mathrm{ec}} \delta_{\mathrm{e} i}$, with $\xi_{\mathrm{ee}}=\sqrt{m_{\mathrm{ve}} / m}<10^{-5}$. Examples with large mixings $\xi$ are given in ref. [5] and will be further discussed elsewhere [11].

The production of heavy Majorana neutrinos proceeds via the process shown in fig. 1 . The kinematics is the 


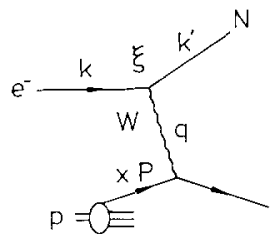

Fig. 1. Production of heavy neutrinos (N) in electron-proton scattering. $\xi$ is the e-N coupling of the charged current.

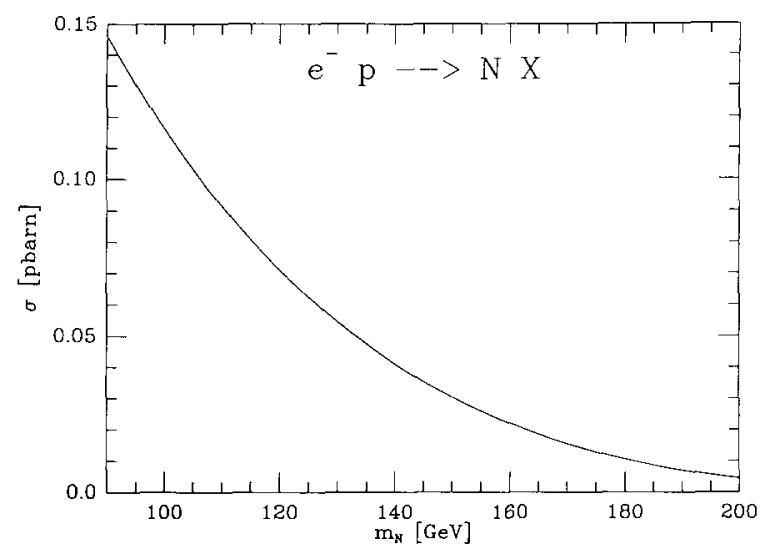

Fig. 2. Total cross section for heavy neutrino production as function of the neutrino mass $m_{\mathrm{N}}\left(\xi^{2}=0.01\right)$.

same as for the production of heavy mirror neutrinos which has been studied in detail by Csikor and Montvay [12] (see also ref. [13]). The usual kinematical variables are

$s=(P+k)^{2}, \quad Q^{2}=-q^{2}, \quad x=\frac{Q^{2}}{2 P \cdot q}, \quad y=\frac{P \cdot q}{P \cdot k}$.

Because of the nonvanishing neutrino mass the variables $x$ and $y$ cannot vary over the entire range from zero to one but are restricted to the intervals

$m_{\mathrm{N}}^{2} / s \leqslant x \leqslant 1, \quad 0 \leqslant y \leqslant 1-m_{\mathrm{N}}^{2} / \hat{s}$,

where $\hat{s}=x s$ is the electron- (anti) quark center of mass energy squared. The cross section differential in $x$ and $y$ for the production of heavy neutrinos is easily calculated. One finds

$\frac{\mathrm{d} \sigma}{\mathrm{d} x \mathrm{~d} y}=\frac{G_{\mathrm{F}}^{2} \xi^{2}}{2 \pi} \frac{m_{\mathrm{W}}^{4}}{\left(y \hat{s}+m_{\mathrm{W}}^{2}\right)^{2}}\left\{\left(\hat{s}-m_{\mathrm{N}}^{2}\right)\left[u\left(x, \mu^{2}\right)+c\left(x, \mu^{2}\right)\right]+(1-y)\left[\hat{s}(1-y)-m_{\mathrm{N}}^{2}\right]\left[d\left(x, \mu^{2}\right)+\bar{s}\left(x, \mu^{2}\right)\right]\right\}$.

Here $G_{\mathrm{F}}$ is Fermi's constant, $\xi$ is the electron-heavy neutrino mixing parameter of the charged current (cf. eq. (9) ), and $u, c, d$ and $s$ are the densities of up, charm, down and strange quarks in the proton. Integration of eq. (12) within the boundaries given by (11) yields the total cross section plotted in fig. 2 , where we have used $\xi^{2}=0.01$ and set 1 of the Duke-Owens densities [14] with scale $\mu \approx 30 \mathrm{GeV}$, which corresponds to the typical transverse momentum of the produced heavy neutrino. The kinematical variables $x$ and $y$ determine longitudinal and transverse momentum of the heavy neutrino (cf. eq. (18)). Integration over the longitudinal momentum yields the transverse momentum spectrum shown in fig. 3 .

The same mixing matrix $\xi$ appears in neutral and charged current (cf. eqs. (8), (9)). Hence, unless the mixing parameters $\xi_{\mathrm{e} i}^{2}$ are very small, the heavy neutrino masses have to be larger than $Z^{0}$-boson mass, i.e. $m_{\mathrm{N}_{i}}>m_{\mathrm{Z}}$. Otherwise decays of the $\mathrm{Z}^{0}$-boson into a light-heavy neutrino pair would have already been observed at LEP. Such heavy neutrinos will predominantly decay into charged lepton and W-boson, with

$\mathrm{BR}\left(\mathrm{N} \rightarrow \ell^{-} \mathrm{W}^{+}\right)=\mathrm{BR}\left(\mathrm{N} \rightarrow \ell^{+} \mathrm{W}^{-}\right)$,

since $\mathrm{N}$ is a Majorana neutrino. The W-boson then decays further, mostly into 2 jets. In the case of large mixing parameters $\xi_{\mathrm{e} i}^{2} \approx 10^{-3}-10^{-2}$, the small upper bounds on light neutrino masses require large off-diagonal ele- 


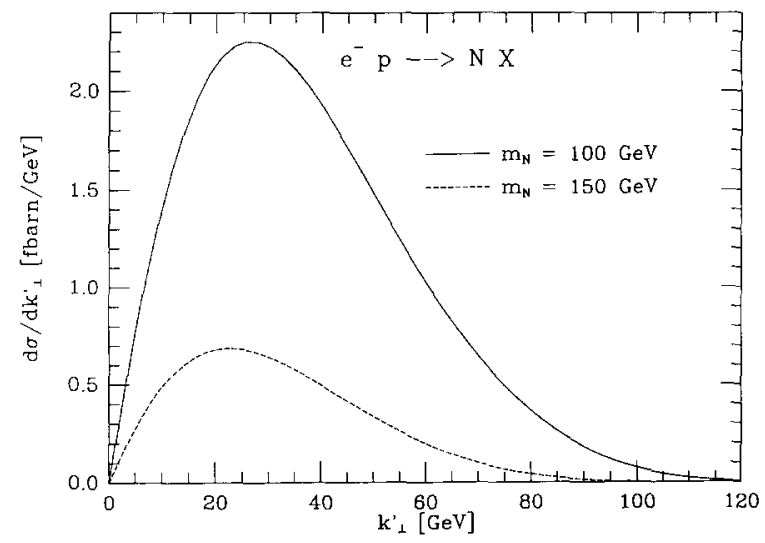

Fig. 3. Transverse momentum spectrum of heavy neutrinos.

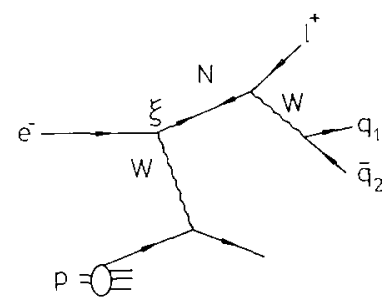

Fig. 4. Production and decay of heavy neutrinos in electron-proton scattering.

ments of $\xi_{\mathrm{e} i}$ (cf. ref. [5]). Hence, we expect the cross sections for the processes $\mathrm{e}^{-} \mathrm{p} \rightarrow \mathrm{e}^{ \pm} \mathrm{W}^{\mp} \mathrm{X}, \mathrm{e}^{-} \mathrm{p} \rightarrow \mu^{ \pm} \mathrm{W}^{\mp} \mathrm{X}$ and $e^{-} p \rightarrow \tau^{ \pm} W^{\mp} X$ to be of similar magnitude.

Let us now evaluate the transverse momentum spectrum of positively charged leptons in the final state. The corresponding Feynman diagram is depicted in fig. 4. Note that this lepton number violating process would be impossible with Dirac neutrinos. The calculation is done in the narrow width approximation with respect to the heavy Majorana neutrino and for an on-shell W-boson. First we work out the differential cross section $\mathrm{d} \sigma /$ $\mathrm{d} x \mathrm{~d} y \mathrm{~d} y_{\ell} \mathrm{d} l_{\perp}$. The kinematic limits for transverse momentum $l_{\perp}$ and rapidity $y_{\ell}$ of the final state lepton are:

$l_{\perp} \leqslant\left[\left(m_{\mathrm{N}}^{2}-m_{\mathrm{W}}^{2}\right) / 2 m_{\mathrm{N}}^{2}\right] \sqrt{s}$,

$\left(m_{\mathrm{N}}^{2}-m_{\mathrm{W}}^{2}-2 l_{\perp} k_{\perp}^{\prime}\right) / 2 m_{\perp} l_{\perp} \leqslant \cosh \left(y_{\mathrm{N}}-y_{\ell}\right) \leqslant\left(m_{\mathrm{N}}^{2}-m_{\mathrm{W}}^{2}+2 l_{\perp} k_{\perp}^{\prime}\right) / 2 m_{\perp} l_{\perp}$.

Here $k_{\perp}^{\prime}, k_{\mathrm{L}}^{\prime}$ are transverse and longitudinal momentum of the heavy neutrino and $y_{\mathrm{N}}$ is its rapidity:

$y_{\mathrm{N}}=\frac{1}{2} \log \left[\left(k^{\prime 0}+k_{\mathrm{L}}^{\prime}\right) /\left(k^{\prime 0}-k_{\mathrm{L}}^{\prime}\right)\right], \quad k^{\prime 0}=\left(m_{\mathrm{N}}^{2}-k_{\perp}^{\prime 2}+k_{\mathrm{L}}^{\prime 2}\right)^{1 / 2}$.

For fixed $l_{\perp}$, eq. (15) implies the lower bound on $k_{\perp}^{\prime}$ :

$k_{\perp}^{\prime} \geqslant k_{\perp}^{\min }=\max \left\{0,\left[4 m_{\mathrm{N}}^{2} l_{\perp}^{2}-\left(m_{\mathrm{N}}^{2}-m_{\mathrm{W}}^{2}\right)^{2}\right] / 4 l_{\perp}\left(m_{\mathrm{N}}^{2}-m_{\mathrm{W}}^{2}\right)\right\}$.

On the other hand, $k_{\perp}^{\prime}$ and $k_{\mathrm{L}}^{\prime}$ are fixed for given $x, y$ and the initial electron and proton energies $E_{\mathrm{e}}$ and $E_{\mathrm{p}}$ :

$k_{\perp}^{\prime}=\left[(1-y) y \hat{s}-y m_{\mathrm{N}}^{2}\right]^{1 / 2}, \quad k_{\mathrm{L}}^{\prime}=x y E_{\mathrm{p}}-(1-y) E_{\mathrm{e}}+m_{\mathrm{N}}^{2} / 4 E_{\mathrm{e}}$.

Eqs. (17) and (18) determine the allowed $(x, y)$ region:

$(1 / s)\left[k_{\perp}^{\prime \min }+m_{\perp}\left(k_{\perp}^{\prime \min }\right)\right]^{2} \leqslant x \leqslant 1$

$\left[\hat{s}-m_{\mathrm{N}}^{2}-\sqrt{\left(\hat{s}-m_{\mathrm{N}}^{2}\right)^{2}-4 \hat{s}\left(k_{\perp}^{\prime \min }\right)^{2}}\right] / 2 \hat{s} \leqslant y \leqslant\left[\hat{s}-m_{\mathrm{N}}^{2}+\sqrt{\left(\hat{s}-m_{\mathrm{N}}^{2}\right)^{2}-4 \hat{s}\left(k_{\perp}^{\prime \min }\right)^{2}}\right] / 2 \hat{s}$,

where $m_{\perp}\left(k_{\perp}^{\prime}\right)=\left(k_{\perp}^{\prime 2}+m_{N}^{2}\right)^{1 / 2}$ is the transverse mass of the heavy neutrino.

A straightforward calculation yields the differential cross section:

$$
\begin{aligned}
& \frac{\mathrm{d} \sigma}{\mathrm{d} x \mathrm{~d} y \mathrm{~d} l_{\perp} \mathrm{d} y_{\ell}}=\frac{G_{\mathrm{F}}^{2} \xi^{2}}{4 \pi^{2} \hat{s}} \frac{m_{\mathrm{W}}^{6}}{\left(y \hat{s}+m_{\mathrm{W}}^{2}\right)^{2}} \frac{m_{\mathrm{N}}^{2} \mathrm{BR}\left(\mathrm{N} \rightarrow \mathrm{e}^{+} \mathrm{W}^{-}\right)}{\left(m_{\mathrm{N}}^{2}-m_{\mathrm{W}}^{2}\right)^{2}\left(m_{\mathrm{N}}^{2}+2 m_{\mathrm{W}}^{2}\right)} \frac{1}{m_{\perp}\left(k_{\perp}^{\prime}\right)} \\
& \quad \times\left(\cosh \left(y_{\mathrm{N}}-y_{\ell}\right)-\frac{m_{\mathrm{N}}^{2}-m_{\mathrm{W}}^{2}-2 l_{\perp} k_{\perp}^{\prime}}{2 m_{\perp} l_{\perp}}\right)^{-1 / 2}\left(\frac{m_{\mathrm{N}}^{2}-m_{\mathrm{W}}^{2}+2 l_{\perp} k_{\perp}^{\prime}}{2 m_{\perp} l_{\perp}}-\cosh \left(y_{\mathrm{N}}-y_{\ell}\right)\right)^{-1 / 2} \\
& \quad \times\left\{A\left[u\left(x, \mu^{2}\right)+c\left(x, \mu^{2}\right)\right]+B\left[d\left(x, \mu^{2}\right)+\bar{s}\left(x, \mu^{2}\right)\right]\right\},
\end{aligned}
$$




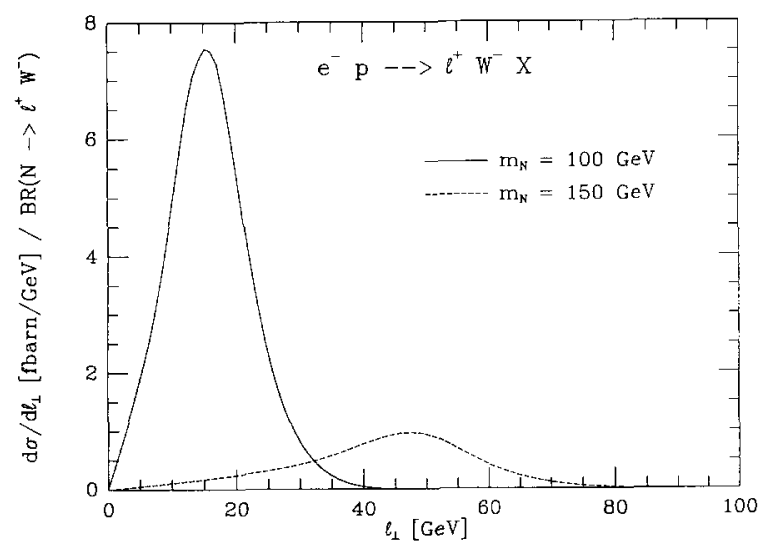

Fig. 5. Transverse momentum spectrum of positively charged leptons in the final state.

$A=\frac{4 \hat{s} m_{\mathrm{N}}^{2}}{m_{\mathrm{W}}^{2}}\left\{\left(\hat{s}-2 m_{\mathrm{W}}^{2}\right)\left(m_{\mathrm{N}}^{2}-m_{\mathrm{W}}^{2}\right)-2\left(m_{\mathrm{N}}^{2}-2 m_{\mathrm{W}}^{2}\right) l_{\perp}\left[E_{\mathrm{e}} \exp \left(y_{\ell}\right)+x E_{\mathrm{p}} \exp \left(-y_{\ell}\right)\right]\right\}$,

$B=\frac{4\left[\hat{s}(1-y)-m_{\mathrm{N}}^{2}\right] m_{\mathrm{N}}^{2}}{m_{\mathrm{W}}^{2}}\left\{\hat{s}(1-y)\left(m_{\mathrm{N}}^{2}-m_{\mathrm{W}}^{2}\right)-2\left(m_{\mathrm{N}}^{2}-2 m_{\mathrm{W}}^{2}\right) x E_{\mathrm{p}} l_{\perp} \exp \left(-y_{\ell}\right)\right\}$,

$\mathrm{BR}\left(\mathrm{N} \rightarrow \mathrm{W}^{-} \mathrm{e}^{+}\right)=\frac{1}{\Gamma_{\mathrm{N}}^{\text {tot }}} \frac{\sqrt{2} G_{\mathrm{F}} \xi^{2}\left(m_{\mathrm{N}}^{2}-m_{\mathrm{W}}^{2}\right)^{2}\left(m_{\mathrm{N}}^{2}+2 m_{\mathrm{W}}^{2}\right)}{16 \pi m_{\mathrm{N}}^{3}}$.

In eq. (23), $\Gamma_{\mathrm{N}}^{\text {tot }}$ denotes the total decay width of the heavy Majorana neutrino $N$. Numerical integration of the variables $y_{\ell}, y$ and $x$ (in this order) over the domains specified in eqs. (15)-(19) yields the transverse momentum spectrum of the final state leptons shown in fig. 5 . Contrary to naive expectations, for $m_{\mathrm{N}}=100 \mathrm{GeV}$ the average transverse momentum of $l^{+}$is not larger than the average transverse momentum of $\mathrm{N}$, which is a kinematical effect due to the relatively small difference between neutrino and W-boson masses.

The decay of a heavy Majorana neutrino is a rather spectacular event, with the constraint that the invariant mass of two jets approximately equals $m_{\mathrm{W}}$. Since there appears to be no significant background, three events should be sufficient to establish the existence of a heavy neutrino. Assuming an integrated luminosity of 200 $\mathrm{pb}^{-1}$ per year at HERA one then obtains from fig. 2 the discovery limits $m_{\mathrm{N}}=180 \mathrm{GeV}$ for $\xi^{2}=0.01$ and $m_{\mathrm{N}}=100$ $\mathrm{GeV}$ for $\xi^{2}=0.001$, which is compatible with the results of Csikor and Montvay [12] for mirror fermions. The transverse momentum spectrum of the final state leptons is very sensitive to the difference between the masses of heavy neutrino and W-boson. The distinctive, qualitative signature of heavy Majorana neutrinos is their equal branching ratio into leptons with positive and negative charge.

We would like to thank F. Eisele, P. Minkowski, I. Montvay and C. Wetterich for stimulating and clarifying discussions.

\section{References}

[1] For a review, see, P. Langacker, in Neutrinos, ed. H.V. Klapdor (Springer, Berlin, 1988) p. 71.

[2] T. Yanagida, Prog. Theor. Phys. B 135 (1978) 66;

M. Gell-Mann et al., in: Supergravity, eds. P. van Nieuwenhuizen and D. Freedman (North-Holland, Amsterdam, 1979).

[3] G. 't Hooft, Phys. Rev. Lett. 37 (1976) 8.

[4] L. Alvarez-Gaumé and E. Witten, Nucl. Phys. B 234 (1983) 269. 
[5] W. Buchmüller and D. Wyler, Phys. Lett. B 249 (1990) 458.

[6] Particle Data Group. J.J. Hernández et al., Review of particle properties, Phys. Lett. B 239 (1990) 1.

[7] R.N. Mohapatra, Phys. Rev. D 34 (1986) 909.

[8] G. Altarelli, B. Mele and R. Rückl, ECFA-CERN report 84-10, Vol. II (1984) p. 549.

[9] R. Eichler, in: Lepton-Photon Symposium (Hamburg 1987), eds. W. Bartel and R. Rückl (North-Holland, Amsterdam, 1988) p. 389.

[10] P. Langacker and D. London, Phys. Rev. D 38 (1988) 886.

[11] W. Buchmüller and C. Greub, in preparation.

[12] F. Csikor and I. Montvay, Phys. Lett. B 231 (1989) 503;

F. Csikor, preprint DESY 90-073 (1990).

[13] F.M.L. Almeida et al., preprint IF/UFRJ/90/23 (1990).

[14] D. Duke and J.F. Owens, Phys. Rev. D 30 (1984) 49. 